\title{
Effects of Saccharomyces boulardii on Neonatal Hyperbilirubinemia: A Randomized Controlled Trial
}

\author{
Ozge Serce, MD ${ }^{1}$ Tugba Gursoy ${ }^{1}$ Fahri Ovali ${ }^{1}$ Guner Karatekin ${ }^{1}$ \\ ${ }^{1}$ Neonatology Unit, Zeynep Kamil Maternity and Children's Research \\ and Training Hospital, Istanbul, Turkey \\ Am J Perinatol 2015;32:137-142. \\ Address for correspondence Ozge Serce, MD, Serce, Neonatalogy \\ Unit, Zeynep Kamil Maternity and Child Health Hospital, Zeynep Kamil \\ Mahallesi. Op. Dr. Burhanettin Ustunel Caddesi No: 10, Uskudar 34668, \\ Istanbul, Turkey (e-mail: ozge_serce@hotmail.com).
}

\begin{abstract}
Keywords

- neonatal hyperbilirubinemia

- phototherapy

- probiotic

- Saccharomyces boulardii

Objective Since probiotics modulate intestinal functions and enterohepatic circulation; they might have an effect on neonatal hyperbilirubinemia treatment. The objective of this study was to investigate the efficacy of Saccharomyces boulardii supplementation on hyperbilirubinemia.

Study Design A prospective, double-blind, placebo controlled trial was performed on 35 to 42 gestational weeks' neonates. They were randomized either to receive feeding supplementation with S. boulardii $125 \mathrm{mg}$ every 12 hours or placebo during phototherapy. Serum bilirubin levels were measured at 0,24 th, 48th, $72 \mathrm{nd}$, and 96th hour of phototherapy.

Results A total of 119 infants (61 in the control group and 58 in the study group) were enrolled. The duration of phototherapy (2 [1-3] vs. 2 [1-3], p: 0.22) was not different between groups. The levels of bilirubin during phototherapy ([24th hour; 14.1 \{12.8-15.7\} vs. 13.5 [12.4-14.9], $p: 0.085]$; [48th hour; 14.1 [12-15.3] vs. 13.4 [12.4-14.5], p: 0.41$]$; [72nd hour; 13.9 \{12.2-15.6\} vs. 13.5 \{12.5-14.5], p: 0.41$]$; [96th hour; $14.7\{11.4-15.5]$ vs. $13.4\{10.7-14.1\}, p: 0.24]$ ) or the duration of rebound phototherapy (1 [1-1] vs. $1.5[1-2], p: 0.40)$ were lower in the study group than in the controls, but none of the values were statistically significant.

Conclusion S. boulardii did not influence the clinical course of hyperbilirubinemia significantly.
\end{abstract}

Severe hyperbilirubinemia continues to be the most common cause of neonatal readmission to hospitals despite attempts to identify newborns at risk of clinically important hyperbilirubinemia before they are discharged from hospital. ${ }^{1-4}$ Recently, increase in early hospital discharges, coupled with a rise in breastfeeding rates, have led to a rise in the rate of hyperbilirubinemia and kernicterus. ${ }^{5,6}$ Also, neonatal hyperbilirubinemia can generate parental anxiety and an increase in hospital costs related to hospital readmissions. ${ }^{7,8}$

received

February 6, 2014

accepted after revision

April 3, 2014

published online

June 10, 2014
Probiotics are viable microorganisms that have beneficial effects in the prevention and treatment of specific pathological conditions. ${ }^{9}$ Probiotic strains can reduce intestinal transit time, improve the quality of regularly occurring muscular contractions in the small intestine, and increase the rate of mitosis in enterocytes, and so decrease enterohepatic circulation. ${ }^{10-14}$ The effect of probiotics on the course of indirect hyperbilirubinemia is a new area for researchers and clinicians. If their possible effects on the bilirubin level or on the phototherapy duration can be confirmed, they may be used

Copyright $\odot 2015$ by Thieme Medical Publishers, Inc., 333 Seventh Avenue, New York, NY 10001, USA.

DOI http://dx.doi.org/ 10.1055/s-0034-1376390. ISSN 0735-1631. 
for prophylaxis of hyperbilirubinemia in the high-risk group. On the other hand, if they influence the duration of phototherapy, they may help decreasing the hospital costs. Since there are no randomized controlled trials on this subject, probiotics at present are not advised for prophylaxis or the treatment of neonatal hyperbilirubinemia. In this study, we aimed to investigate the effect of orally administered Saccharomyces boulardii, probiotic yeast, on the duration of phototherapy and on the course of serum bilirubin levels during phototherapy in neonates.

\section{Patients and Methods}

We performed this prospective, masked, randomized, controlled trial between July and August 2013, in Zeynep Kamil Maternity and Children's Research and Training Hospital. Local ethical committee approved the study. Neonates, born in 35 to 42 gestational weeks, who need phototherapy for neonatal hyperbilirubinemia, were eligible for the trial. Infants who had asphyxia, major congenital anomalies, direct coombs test positive or negative $\mathrm{ABO} / \mathrm{Rh}$ incompatibility, hypothyroidism, gastrointestinal obstruction, inborn errors of metabolism, cephalohematoma or infection were excluded. Infants who received formula were dropped out from the study due to their bilirubin reducing effect.

With an $\alpha$ error $=0.05$ and $\beta$ error $=0.8$, a minimum of 31 infants per group were required to detect a reduction in the intervention group of at least $1 \mathrm{mg} / \mathrm{dL}$ in the mean bilirubin levels (one-tailed test). The infants enrolled in the study were assigned randomly to the study or control group after informed parental consent was obtained. Randomization was performed by using sequential numbers generated at the computer center. The allocations were contained in opaque, sequentially numbered sealed envelopes. The study group received $S$. boulardii (Reflor, Biocodex, France, $125 \mathrm{mg}$ per dose twice daily); whereas the control group received placebo (distilled water; $1 \mathrm{~mL}$ per dose twice daily) which were added to a small amount of breast milk during phototherapy. Supplementations were started with phototherapy and continued until phototherapy was stopped. The dosage of $S$. boulardii used in this study was empirically decided in accordance with the results of the previous study performed in children. ${ }^{15} \mathrm{~S}$. boulardii was kept in a dry place at room temperature, away from light and humidity, in tightly closed container, and mixed with breast milk before feeding. One milliliter suspension was prepared with mixture of the powder of $S$. boulardii with distilled water. This supplementation did not change the appearance of the milk. Fresh suspension of supplements was prepared by personnel in the breast milk team who were not involved in the care of the neonate and who followed the instructions from the sealed envelope. Thus, the only personnel who knew of the neonates' group assignments were the investigator and those in the breast milk team who were not involved in the care of the neonates.

Demographic and clinical variables of the neonates and adverse events were recorded. The gestational age was estimated based on ultrasound examination before the end of 20 gestational weeks (73\%) or from the last menstrual period. Serum bilirubin concentrations were measured on venous blood samples using Vitros 750 Ortho-Clinical Diagnostics, Johnson and Johnson Company, England, United Kingdom. The measurements were performed at the start time, 24th, 48th, 72nd, 96th hour during and at the end of phototherapy. The duration of phototherapy were monitored.

The Neoblue LED phototherapy system (intensity: $30 \mu \mathrm{W} /$ $\mathrm{cm}^{2} / \mathrm{nm}$, spectrum: 450-470 nm; Natus Medical Inc., San Carlos, CA) was used. The irradiance of the lamps were measured weekly and replaced if necessary. Criteria for starting phototherapy were based on American Academy of Pediatrics (AAP) guidelines, but adapted to the Turkish population. ${ }^{16}$ We accepted all neonates, high-risk neonates as the Turkish people are an Asian race in whom glucose-6phosphate dehydrogenase deficiency is highly frequent. ${ }^{17}$ Phototherapy was stopped when serum bilirubin level was below $2 \mathrm{mg} / \mathrm{dL}$ from the lowest limit for phototherapy.

Statistical analyses were performed by SPSS statistics program v.13.0 (SPSS Inc., Chicago, IL). Data were stated as ratio, mean ( \pm standard deviation), and median (25-75\% percentiles). Mann-Whitney $U$ test was used to compare continuous variables and the chi-square test was used to compare categorical variables. A $p$ value of $\leq 0.05$ was considered statistically significant.

\section{Results}

There were 255 neonates admitted to our neonatal unit for phototherapy during the study period. A total of 122 neonates were excluded, due to family members declined consent for the study $(n=14)$, blood group incompatibility $(n=87)$, severe asphyxia $(n=2)$, major congenital anomaly $(n=3)$, inborn errors of metabolism $(n=3)$, cephalohematoma $(n=9)$, or proven infection was $(n=4)$. A total of 119 neonates were enrolled in the trial; 14 neonates in study group and 15 neonates in control group dropped out of the study because of discontinued intervention, formula feeding or family withdrawal. Finally, 61 infants in the control group, 58 infants in the study group completed the study.

Characteristics of the study population are described in -Table 1. - Table 2 shows the phototherapy duration and bilirubin levels of the neonates. The median starting time of phototherapy was at postnatal 2nd day in both of the groups (-Table $\mathbf{1}$ ). The duration of phototherapy was not different between groups. Either the levels of bilirubin during phototherapy or the rebound phototherapy duration were lower in the study group than in the controls, but all values were not statistically significant (-Table $\mathbf{2}$ ).

The rate of decline of bilirubin and the duration of phototherapy is shown in - Fig. 1.

We did not observe any side effects (gastrointestinal intolerance; higher osmotic load causing abdominal distension, diarrhea or vomiting and sepsis due to probiotics) during the study.

\section{Discussion}

Factors affecting the metabolism of bilirubin in newborns may change the risk of bilirubin induced encephalopathy or 
Table 1 Characteristics of the neonates

\begin{tabular}{|l|l|l|l|}
\hline & Control group $(\boldsymbol{n}=\mathbf{6 1})$ & Study group $(\boldsymbol{n}=\mathbf{5 8})$ & $p$-Value \\
\hline Maternal age (y), mean \pm SD & $27.2 \pm 5.6$ & $27.5 \pm 5.3$ & 0.69 \\
\hline Primiparity, $n(\%)$ & $23(37.7)$ & $39(67.2)$ & 0.001 \\
\hline Gestational age (wk), mean \pm SD & $37.9 \pm 2.02$ & $37.7+1.7$ & 0.48 \\
\hline Birth weight (g), mean \pm SD & $3,223 \pm 609$ & $3,071 \pm 593$ & 0.17 \\
\hline Male, $n$ (\%) & $29(47.5)$ & $26(44.8)$ & 0.76 \\
\hline Diabetic mother, $n(\%)$ & $2(3)$ & $3(5)$ & 0.28 \\
\hline Preeclampsia, $n(\%)$ & $3(5.2)$ & 0.35 \\
\hline Postnatal age, median (25-75\%) & $1(1.6)$ & $2(1-3)$ & 0.90 \\
\hline
\end{tabular}

Table 2 Phototherapy duration and bilirubin levels of the neonates

\begin{tabular}{|c|c|c|c|}
\hline & $\begin{array}{l}\text { Control group } \\
(n=61)\end{array}$ & $\begin{array}{l}\text { Study group } \\
(n=58)\end{array}$ & $p$-Value \\
\hline \multicolumn{4}{|c|}{ Total bilirubin level during phototherapy, mg/dL, median (25-75\%) } \\
\hline $0 \mathrm{~h}$ & $13.5(12-15.2)$ & $14(11.5-15.4)$ & 0.60 \\
\hline $24 \mathrm{~h}$ & $14.1(12.8-15.7)$ & $13.5(12.4-14.9)$ & 0.085 \\
\hline $48 \mathrm{~h}$ & $14.1(12-15.3)$ & $13.4(12.4-14.5)$ & 0.41 \\
\hline $72 \mathrm{~h}$ & $13.9(12.2-15.6)$ & $13.5(12.5-14.5)$ & 0.41 \\
\hline $96 \mathrm{~h}$ & $14.7(11.4-15.5)$ & $13.4(10.7-14.1)$ & 0.24 \\
\hline Phototherapy duration, median (25-75\%) & $2(1-3)$ & $2(1-3)$ & 0.22 \\
\hline Rebound hyperbilirubinemia, $n(\%)$ & $3(4.9)$ & $6(10.3)$ & 0.31 \\
\hline Rebound phototherapy duration, median (25-75\%) & $1.5(1-2)$ & $1(1-1)$ & 0.40 \\
\hline
\end{tabular}

kernicterus. Displacement of bilirubin from albumin, inducing uridine diphosphate glucuronyl-transferase (UDGP-T) by drugs and enhancement of enterohepatic circulation by pathologic conditions may increase bilirubin toxicity. ${ }^{18}$ Therefore, the main treatment modalities of hyperbilirubinemia are based on increasing bilirubin conjugation, decreasing enterohepatic circulation, inhibiting bilirubin production, and hemolysis. ${ }^{18}$

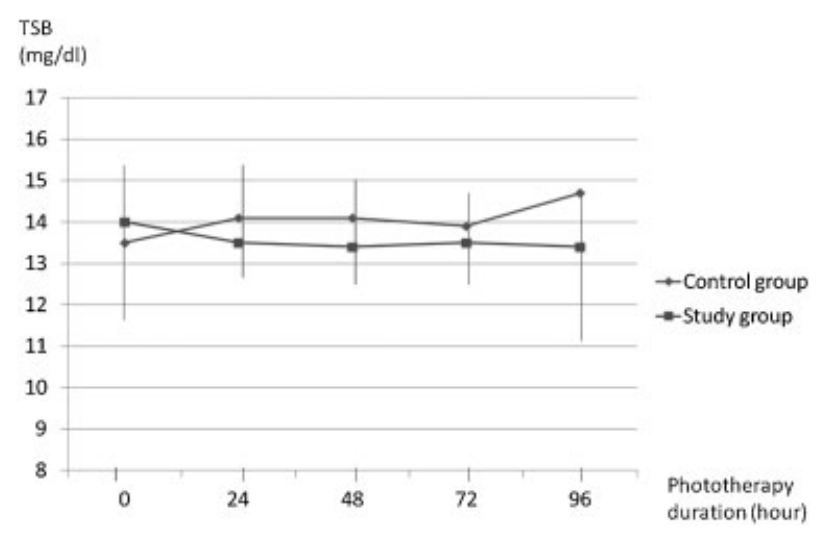

Fig. 1 Bilirubin levels during the study period.
At birth, the neonatal intestinal tract is virtually sterile. As the human intestine is naturally exposed to a contaminated bacterial environment, the bowel becomes colonized quickly after birth with a variety of bacterial species. Supporting colonization of gastrointestinal tract by enteral supplementation with probiotic microorganisms, which inhibits pathogenic bacteria at intestinal flora potentially provides benefit to the host. ${ }^{19}$ Probiotics increase intestinal transit time and suppress reabsorption of bile acids into the enterohepatic circulation of bilirubin. ${ }^{10,11}$ It is important to note that there are many different mechanisms producing the benefits of probiotics and there are also strain-specific effects. ${ }^{20}$ Furthermore, there is no clear suggestion in the literature about the optimal dose, length of treatment, and strain of probiotic bacteria that should be administered to neonates. Choosing which probiotics to use, therefore, is a challenge. At present, limited clinical trials which were performed in local hospitals of China that prefer to investigate bacterial strains demonstrated the positive effect probiotics on the prophylaxis or treatment of hyperbilirubinemia in term neonates. ${ }^{21,22}$ On the other hand, the clinical trials' design was highly heterogenic. There is limited data on the effects of $S$. boulardii in term and preterm infants. Therefore, we preferred to investigate $S$. boulardii which is a yeast, probiotic, and polyamine producer although this effect was shown in very low birth 
weight infants at the trial of Demirel et al. ${ }^{15,23,24}$ Although this study indicates that $S$. boulardii supplementation is effective in reducing the duration of phototherapy, it has no effect on total bilirubin levels at the beginning and end of the phototherapy treatment. Therefore, we may say that $S$. boulardii may be more effective than other probiotics on the course of hyperbilirubinemia due to enhancement of intestinal maturation by the effect on polyamines which are essential for cell growth and differentiation. ${ }^{15}$

Our data demonstrates the beneficial effect of $S$. boulardii on the duration of rebound phototherapy. It lowered the serum bilirubin levels during phototherapy, but these results were not statistically significant. Also, we did not observe any positive effect on the rate of rebound hyperbilirubinemia. Demirel et al explained the beneficial effect of $S$. boulardii by its improving effect on the feeding intolerance and suppressing effect on the resorption of bilirubin into the enterohepatic circulation. ${ }^{23}$ During the interdigestive period, term neonates exhibit multiphasic cycles of intestinal motor activity, which are termed migratory motor complex. Physiologic studies have shown a lack of the propagative phase III of the migratory motor complex in the duodenum of preterm neonates less than 32 weeks gestational age. ${ }^{25}$ So, feeding intolerance is a common problem in preterm neonates in contrast to term neonates. This physiologic dissimilarity between preterm and term neonates may explain why our results differ from Demirel et al's study. Since the number of studies about probiotics on this area is small, we preferred to investigate a single strain preparation. When the exact effect of single strain probiotics are shown, future studies with combined preparations with different probiotic microorganisms and also with synbiotics should be planned.

There must be an optimal dose for "viability," which means the ability of the probiotic strain to survive and proliferate in "adequate" numbers after overcoming the barriers such as gastric acid, bile, and competing flora to have beneficial effects on the host. ${ }^{20}$ So, the empiric dose we used or starting supplementation of probiotic with phototherapy may be questioned in our study as the dose might be inadequate or the timing for the treatment might be too late for optimal colonization. Postnatal supplementation of probiotics as soon as possible should also be investigated before making any conclusion that $S$. boulardii is not effective on the course of hyperbilirubinemia.

In conclusion, $S$. boulardii is still not a choice for adjuvant treatment of neonatal hyperbilirubinemia. Large randomized controlled studies, designed with standard protocols with different probiotic strains, which observe clinical outcomes with fecal flora analyses on this area are required.

\section{Note}

The full trial protocol can be accessed where the trial was approved (Central ethical committee in Ankara, Turkey). This trial was registered by IRCT with ID as IRCT 2012123010279 N2.

\author{
Competing Interest \\ The drugs were supplied by Biocodex.
}

\section{References}

1 Sgro M, Campbell D, Shah V. Incidence and causes of severe neonatal hyperbilirubinemia in Canada. CMAJ 2006;175(6):587-590

2 Bhutani VK, Johnson LH. Urgent clinical need for accurate and precise bilirubin measurements in the United States to prevent kernicterus. Clin Chem 2004;50(3):477-480

3 Bhutani VK, Johnson LH, Keren R. Diagnosis and management of hyperbilirubinemia in the term neonate: for a safer first week. Pediatr Clin North Am 2004;51(4):843-861, vii

4 Bhutani VK, Johnson L, Sivieri EM. Predictive ability of a predischarge hour-specific serum bilirubin for subsequent significant hyperbilirubinemia in healthy term and near-term newborns. Pediatrics 1999;103(1):6-14

5 Ip S, Chung M, Kulig J, et al; American Academy of Pediatrics Subcommittee on Hyperbilirubinemia. An evidence-based review of important issues concerning neonatal hyperbilirubinemia. Pediatrics 2004;114(1):e130-e153

6 Joint Commission on Accreditation of Healthcare Organizations. Kernicterus threatens healthy newborns. Sentinel Event Alert 2001;(18):1-4

7 Bisceglia M, Indrio F, Riezzo G, Poerio V, Corapi U, Raimondi F. The effect of prebiotics in the management of neonatal hyperbilirubinaemia. Acta Paediatr 2009;98(10):1579-1581

8 Burke BL, Robbins JM, Bird TM, Hobbs CA, Nesmith C, Tilford JM. Trends in hospitalizations for neonatal jaundice and kernicterus in the United States, 1988-2005. Pediatrics 2009;123(2):524-532

9 Havenaar R, Huis in't Veld JHJ. Probiotics: a general view. In: Wood $\mathrm{BJB}$, ed. The Lactic Acid Bacteria in Health and Disease. The Lactic Acid Bacteria Vol. 1. New York, NY: Chapman and Hall; 1992:209-224

10 Husebye E, Hellström PM, Sundler F, Chen J, Midtvedt T. Influence of microbial species on small intestinal myoelectric activity and transit in germ-free rats. Am J Physiol Gastrointest Liver Physiol 2001;280(3):G368-G380

11 Banasaz M, Norin E, Holma R, Midtvedt T. Increased enterocyte production in gnotobiotic rats mono-associated with Lactobacillus rhamnosus GG. Appl Environ Microbiol 2002;68(6):3031-3034

12 van Meer H, Boehm G, Stellaard F, et al. Prebiotic oligosaccharides and the enterohepatic circulation of bile salts in rats. Am J Physiol Gastrointest Liver Physiol 2008;294(2):G540-G547

13 Floch MH. Bile salts, intestinal microflora and enterohepatic circulation. Dig Liver Dis 2002;34(Suppl 2):S54-S57

14 Usman HA, Hosono A. Effect of administration of Lactobacillus gasseri on serum lipids and fecal steroids in hypercholesterolemic rats. J Dairy Sci 2000;83(8):1705-1711

15 Costalos C, Skouteri V, Gounaris A, et al. Enteral feeding of premature infants with Saccharomyces boulardii. Early Hum Dev 2003;74(2):89-96

16 American Academy of Pediatrics Subcommittee on Hyperbilirubinemia. Management of hyperbilirubinemia in the newborn infant 35 or more weeks of gestation. Pediatrics 2004;114(1):297-316

17 Kaplan M, Merlob P, Regev R. Israel guidelines for the management of neonatal hyperbilirubinemia and prevention of kernicterus. J Perinatol 2008;28(6):389-397

18 Blackburn S. Hyperbilirubinemia and neonatal jaundice. Neonatal Netw 1995;14(7):15-25

19 Bin-Nun A, Bromiker R, Wilschanski M, et al. Oral probiotics prevent necrotizing enterocolitis in very low birth weight neonates. J Pediatr 2005;147(2):192-196

20 Deshpande GC, Rao SC, Keil AD, Patole SK. Evidence-based guidelines for use of probiotics in preterm neonates. BMC Med 2011;9:92

21 Wang WJ, Zheng YJ. Clinical application of probotics in China. Chin J Microecol 2009-01 
22 Liu L, Bao LY, Zhang L, Cui YL. The observation on effect of Clostridium butyricum powder in treatment of breast milk jaundice. Chin J Microecol 2009;21(3):260-261

23 Demirel G, Celik IH, Erdeve O, Dilmen U. Impact of probiotics on the course of indirect hyperbilirubinemia and phototherapy duration in very low birth weight infants. J Matern Fetal Neonatal Med 2013;26(2):215-218
24 Serce O, Benzer D, Gursoy T, Karatekin G, Ovali F. Efficacy of saccharomyces boulardii on necrotizing enterocolitis or sepsis in very low birth weight infants: a randomised controlled trial. Early Hum Dev 2013;89(12):1033-1036

$25 \mathrm{Ng}$ E, Shah VS. Erythromycin for the prevention and treatment of feeding intolerance in preterm infants. Cochrane Database Syst Rev 2008;16(3):CD001815

Figure 1 of this article has been corrected as per the online erratum published on August 14, 2014.

The DOI of the erratum is $10.1055 / \mathrm{s}-0034-1389078$. 